\title{
Observations of warm molecular gas and kinematics in the disc around HD 100546
}

\author{
O. Panić ${ }^{1,2}$, E. F. van Dishoeck ${ }^{1,3}$, M. R. Hogerheijde ${ }^{1}$, A. Belloche ${ }^{4}$, R. Güsten ${ }^{4}$, W. Boland ${ }^{1,5}$, and A. Baryshev ${ }^{6}$ \\ ${ }^{1}$ Leiden Observatory, Leiden University, PO Box 9513, 2300 RA Leiden, The Netherlands \\ 2 European Southern Observatory, Karl Schwarzschild str. 2, 85748 Garching, Germany \\ e-mail: opanic@eso.org \\ 3 Max-Planck-Institut für extraterrestrische Physik, Postfach 1312, 85741 Garching, Germany \\ ${ }^{4}$ Max-Planck Institut für Radioastronomie, Auf dem Hügel 69, 53121 Bonn, Germany \\ 5 Nederlandse Onderzoeksschool Voor Astronomie (NOVA), PO Box 9513, 2300 RA Leiden, The Netherlands \\ ${ }^{6}$ SRON Netherlands Institute for Space Research, PO Box 800, 9700 AV Groningen, The Netherlands
}

Received 15 January 2010 / Accepted 3 May 2010

\section{ABSTRACT}

\begin{abstract}
Context. The disc around the Herbig Ae/Be star HD 100546 is one of the most extensively studied discs in the southern sky. Although there is a wealth of information about its dust content and composition, not much is known about its gas and large-scale kinematics. Many recent results have stressed the importance of studying both the gas and dust in discs. ${ }^{12} \mathrm{CO}$ is an excellent gas tracer in the submillimetre, and the intensity ratio between lines originating from low and high rotational levels probes the gas temperature. Emerging submillimetre facilities in the Southern hemisphere allow us to characterise the gas and dust content in objects like HD 100546 better. Aims. Our aim is to establish whether the disc is gas-rich and to study the disc temperature and kinematics.

Methods. We detected and studied the molecular gas in the disc at spatial resolution from 7'.7 to $18^{\prime \prime} .9$ using the Atacama Pathfinder Experiment telescope ${ }^{\star}$. We observed the lines ${ }^{12} \mathrm{CO} J=7-6, J=6-5, J=3-2,{ }^{13} \mathrm{CO} J=3-2$ and $[\mathrm{C} \mathrm{I}]{ }^{3} \mathrm{P}_{2}-{ }^{3} \mathrm{P}_{1}$, as diagnostics of disc temperature, size, chemistry, and kinematics. We use parametric disc models that reproduce the low- $J^{12} \mathrm{CO}$ emission from Herbig Ae stars and we vary the basic disc parameters - temperature, mass, and size. With the help of a molecular excitation and radiative transfer code we fit the observed spectral line profiles.

Results. Our observations are consistent with more than $10^{-3} M_{\odot}$ of molecular gas in a disc of $\approx 400$ AU radius in Keplerian rotation around a $2.5 M_{\odot}$ star, seen at an inclination of $50^{\circ}$. The detected ${ }^{12} \mathrm{CO}$ lines are dominated by gas at $30-70 \mathrm{~K}$. Not detecting the [C I] line indicates excess ultraviolet emission above that of a B9 type model stellar atmosphere. Asymmetry in the ${ }^{12} \mathrm{CO}$ line emission suggests that one side of the outer disc is colder by $10-20 \mathrm{~K}$ than the other. A plausible scenario is a warped geometry in the inner disc casting a partial shaddow on the outer disc. We exclude pointing offsets, foreground cloud absorption, and asymmetry in the disc extent as possible causes of the observed line asymmetry.

Conclusions. Efficient heating of the outer disc by the star HD 100546 ensures that low- and high- $J{ }^{12} \mathrm{CO}$ lines are dominated by the outermost disc regions, indicating a $400 \mathrm{AU}$ radius. The ${ }^{12} \mathrm{CO} J=6-5$ line arises from a disc layer higher than disc midplane, and warmer by $15-20 \mathrm{~K}$ than the layer emitting the $J=3-2$ line. The existing models of discs around Herbig Ae stars, asuming a B9.5 type model stellar atmosphere, overproduce the $[\mathrm{CI}]{ }^{3} \mathrm{P}_{2}-{ }^{3} \mathrm{P}_{1}$ line intensity from HD 100546 by an order of magnitude.
\end{abstract}

Key words. planetary systems: protoplanetary disks - stars: pre-main sequence - ISM: molecules

\section{Introduction}

Over the past decade our understanding of the structural and physical properties of discs around young stars has increased from basic theoretical modelling of the spectral energy distributions (SEDs) constrained by observations with no spatial information to modelling based not only on the SEDs, but also on spatially resolved dust observations, such as scattered light images and interferometry (Pinte et al. 2008; Panić et al. 2008; Tannirkulam et al. 2008). Two decades ago, the first submillimetre interferometer observations resolved the molecular gas emission spatially, and this allowed major progress in understanding the disc kinematics, structure, and chemistry (e.g., Beckwith \& Sargent 1987; Koerner et al. 1993; Dutrey et al. 1994). A more recent example is a study of one of the brightest discs around a Herbig Ae star, HD 163296, shown in Isella et al. (2007).

* This publication is based on data acquired with the Atacama Pathfinder Experiment (APEX). APEX is a collaboration between the Max-Planck-Institut für Radioastronomie, the European Southern Observatory, and the Onsala Space Observatory.
(Sub)millimetre gas and dust emission is the ideal probe of the global disc properties, such as size, mass, and radial distribution of disc material, because the bulk of the disc mass is located beyond $100 \mathrm{AU}$ from the star, at temperatures of $10-50 \mathrm{~K}$ that dominate this part of the spectrum. Disc models that include constraints on both dust and molecular gas observations have stressed the importance of analysing the gas and dust components simultaneously, in the context of a single disc model with a three-dimensional temperature and density structure (Wilner et al. 2003; Raman et al. 2006; Panić et al. 2008, 2009).

Until recently, observations of rotational transitions of molecules in the submillimetre regime have been focused primarily on the low- $J$ emission from ${ }^{12} \mathrm{CO}$, up to the $J=3-2$ line (Greaves et al. 2000; Thi et al. 2001; Qi et al. 2004; Thi et al. 2004; Dent et al. 2005). In two of the brightest and most studied sources, TW Hya and LkCa 15, the observations of higher- $J$ transitions of ${ }^{12} \mathrm{CO}$, up to $J=6-5\left(E_{\mathrm{k}}=116 \mathrm{~K}\right)$, have been compared to the low- $J$ lines, providing estimates of the gas temperature in the intermediate-height molecular layer (van Zadelhoff et al. 2001a), crucial ingredients for chemical modelling of discs. 
These single-dish line spectra were fitted using simplistic disc models, deriving a temperature of $20-40 \mathrm{~K}$ in the ${ }^{12} \mathrm{CO}$ line emitting layers of $\mathrm{LkCa} 15$, and more than $40 \mathrm{~K}$ in TW Hya. Qi et al. (2006) analysed submillimetric interferometer observations of TW Hya in the context of a disc structure based on an accretion disc model (Calvet et al. 2002). Based on ${ }^{12} \mathrm{CO} J=6-5$, 3-2 and 2-1 observations, they show that X-ray heating of the gas is efficient in this source, in addition to the stellar radiation field. Such diagnostics of gas heating and ionisation improve our understanding of how the gas content evolves in discs.

The emerging (sub)millimetre facilities in the Southern hemisphere like the Atacama Pathfinder EXperiment (APEX) and the Australia Telescope Compact Array (ATCA) are opening a window towards the star-forming regions of the Southern sky and are well suited to studies of circumstellar disc emission. These instruments also pave the way for future observations with the Atacama Large Millimetre/Submillimeter Array (ALMA), which will drastically improve our knowledge of disc structure and evolution (e.g. Guilloteau \& Dutrey 2008). We use APEX receivers $\mathrm{APEX}-2 \mathrm{a}$ and $\mathrm{CHAMP}^{+}$to observe the ${ }^{12} \mathrm{CO} J=$ $7-6, J=6-5, J=3-2,{ }^{13} \mathrm{CO} J=3-2$ and $[\mathrm{C} \mathrm{I}]{ }^{3} \mathrm{P}_{2}-{ }^{3} \mathrm{P}_{1}$ line emission towards the disc around the young intermediatemass star HD 100546. A wealth of observations of dust in this bright disc (Waelkens et al. 1996; Malfait et al. 1998; Grady et al. 2001; Augereau et al. 2001; Bouwman et al. 2003; Acke \& van den Ancker 2006a; Ardila et al. 2007) has motivated us to probe its molecular gas content and kinematics. The chosen transitions are particularly sensitive to the gas in the warm upper layers and kinematics of the outer disc. Our millimetre line observations probe the outer radius and inclination. The existing observational constraints on these parameters in the disc around HD 100546 provide an excellent basis for the analysis of our data. Our observations also provide a bridge toward even higher- $J$ far infrared ${ }^{12} \mathrm{CO}$ transitions to be observed with the Herschel Space Observatory. HD 100546 is a young B9V type, $2.5 M_{\odot}$ star, classified as a Herbig Be star because of its isolation, infrared excess, and silicate emission (Thé et al. 1994; Malfait et al. 1998). With a distance of $103 \pm 6 \mathrm{pc}$, measured by Hipparcos, this is one of the nearest Herbig Ae/Be stars. The age of the star is estimated to be greater than $10 \mathrm{Myr}$ (van den Ancker et al. 1998). This makes the presence of circumstellar material intriguing, considering that discs are found to dissipate within $10 \mathrm{Myr}$ in most young stars (e.g., Hollenbach et al. 2000; Hernández et al. 2007; Hillenbrand 2008). Based on SED modelling, Bouwman et al. (2003) postulate there is an inner hole in the disc with a 10 AU radius, possibly caused by a Jupiter-sized planet (see also Acke \& van den Ancker 2006a). Direct evidence of cold disc material at larger radii is provided by ATCA observations of Wilner et al. (2003) at $89 \mathrm{GHz}$ $(3.4 \mathrm{~mm})$ and $2^{\prime \prime}$ resolution, with the flux of $36 \pm 3 \mathrm{mJy}$, values consistent with the $1.3 \mathrm{~mm}$ observations of Henning et al. (1998). They do not detect $\mathrm{HCO}^{+} J=1-0$ line emission and speculate that photodissociation of its progenitor species, ${ }^{12} \mathrm{CO}$, in the upper disc layers or an overall gas depletion may be the reason for this. Recent spectroastrometric observations of rovibrational ${ }^{12} \mathrm{CO}$ transitions by van der Plas et al. (2009) suggest that this molecular species is missing from the inner disc, at least up to $8 \mathrm{AU}$ from the star (also see Brittain et al. 2009).

Scattered light imaging of HD 100546 reveals the disc extending up to $4^{\prime \prime}$ from the star viewed at an inclination of $50^{\circ}$ and an interesting disc structure resembling spiral arms (Pantin et al. 2000; Augereau et al. 2001; Grady et al. 2001). This structure was interpreted as the result of disc perturbation by a companion (Quillen et al. 2005) or a warped disc structure (Quillen 2006).
Coronographic imaging by Augereau et al. (2001) shows steep surface brightness profiles in the environment of HD 100546 indicative of optically thin emission in the near-infrared, with surface densities as low as $10^{-3} \mathrm{~g} \mathrm{~cm}^{-2}$. Their images trace the emission of small dust $(<5 \mu \mathrm{m})$, extending out to $800 \mathrm{AU}$ from the star. The authors suggest there are an optically thick disc with a 400 AU radius and an optically thin flattened halo or envelope farther from the star. The scattered light images hint at the presence of gas in the disc that supports the disc vertical structure.

In this work, we detect and study the molecular gas in the disc, in particular its kinematics and temperature, at spatial resolution from 7'.7 to $18^{\prime \prime}$.9. In Sect. 2 we present our observations of ${ }^{12} \mathrm{CO},{ }^{13} \mathrm{CO}$, and [C I] lines. All ${ }^{12} \mathrm{CO}$ lines are detected, there is a tentative detection of the ${ }^{13} \mathrm{CO}$ line, while [C I] emission is not detected. We model the spectra in Sect. 3 discussing the implications for the disc size, mass, and kinematics. We identify the regions dominating the observed lines, and derive their temperatures. Section 4 summarises our results.

\section{Observations and results}

The observations of ${ }^{12} \mathrm{CO} J=6-5$ at $691.472 \mathrm{GHz}$ and [C I] ${ }^{3} \mathrm{P}_{2}-{ }^{3} \mathrm{P}_{1}$ ([C I] $J=2-1$ hereafter) at $809.344 \mathrm{GHz}$ towards HD 100546 at $\mathrm{RA}=11^{\mathrm{h}} 33^{\mathrm{m}} 25^{\mathrm{s}} .4$ and $\mathrm{Dec}=-70^{\circ} 11^{\prime} 41^{\prime \prime}$ (J2000) were obtained simultaneously, using the $\mathrm{CHAMP}^{+}$heterodyne array receiver (Güsten et al. 2006) on APEX on 2008 November 11 . The 7 pixels in each wavelength band are arranged in a hexagon of 6 pixels around one central pixel pointed towards the source, with beam sizes of $9^{\prime \prime}$ at $691 \mathrm{GHz}$ and 7.'7 at $809 \mathrm{GHz}$. The data were obtained in the position switching mode in which the telescope moved between the on-source position and a reference position at an equatorial offset of $\left(-1000^{\prime \prime}, 0^{\prime \prime}\right)$ from the source. The backend consisting of the Fast Fourier Transform Spectrometer unit was used on all pixels, providing a spectral resolution of $0.37 \mathrm{MHz}$ or $0.14-0.16 \mathrm{~km} \mathrm{~s}^{-1}$ at these frequencies, and covering a bandwidth of $1.5 \mathrm{GHz}$ or 4096 channels. Main beam efficiencies are 0.40 at $691 \mathrm{GHz}$ and 0.37 at $809 \mathrm{GHz}$. Calibration is uncertain by $\approx 30 \%$ at both frequencies. Pointing was performed directly prior to and after the on-source integration providing an accuracy better than $3^{\prime \prime}$. The pointing source was $07454-7112$, about $20^{\circ}$ away from HD 100546 , at the same airmass.

The CO $J=6-5$ line was also observed on 2008 November 10 in jiggle mode and its intensity and spectral profile were found to be the same within $20 \%$, as in the 2008 November 11 data. Since these data are noisier, the data sets taken on different dates were not combined. During this latter observation, the high band was tuned to ${ }^{12} \mathrm{CO} J=7-6$ at $806.665 \mathrm{GHz}$, with the beam efficiency of 0.37 . In the further analysis, we used only the high $\mathrm{S} / \mathrm{N}{ }^{12} \mathrm{CO} J=6-5$ data that were taken on 2008 November 11 . The ${ }^{12} \mathrm{CO} J=3-2$ line at $345.796 \mathrm{GHz}$ and the ${ }^{13} \mathrm{CO} J=3-2$ line at $330.588 \mathrm{GHz}$ were observed on 2005 July 27 and 28 with the APEX-2a receiver using a single pointing. The channel spacing of these data is $61 \mathrm{kHz}$ or $0.05-0.06 \mathrm{~km} \mathrm{~s}^{-1}$, and the spectral resolution is $98 \mathrm{kHz}$ or $0.09 \mathrm{~km} \mathrm{~s}^{-1}$ (Klein et al. 2006). The main beam efficiency of APEX at $346 \mathrm{GHz}$ is 0.73 , and the beam sizes are $18^{\prime \prime} .1$ at $345.796 \mathrm{GHz}$ and $18^{\prime \prime} .9$ at $330.588 \mathrm{GHz}$, respectively. Calibration is uncertain by $\approx 20 \%$ at these frequencies. Our ${ }^{12} \mathrm{CO} J=3-2$ line data were presented in Panić \& Hogerheijde (2009). In all our observations, APEX forward efficiency of 0.95 was taken into account.

The data were reduced and analysed using the CLASS and STARLINK software. We detect all the observed ${ }^{12} \mathrm{CO}$ line 
O. Panić et al.: Warm molecular gas and kinematics in the disc around HD 100546

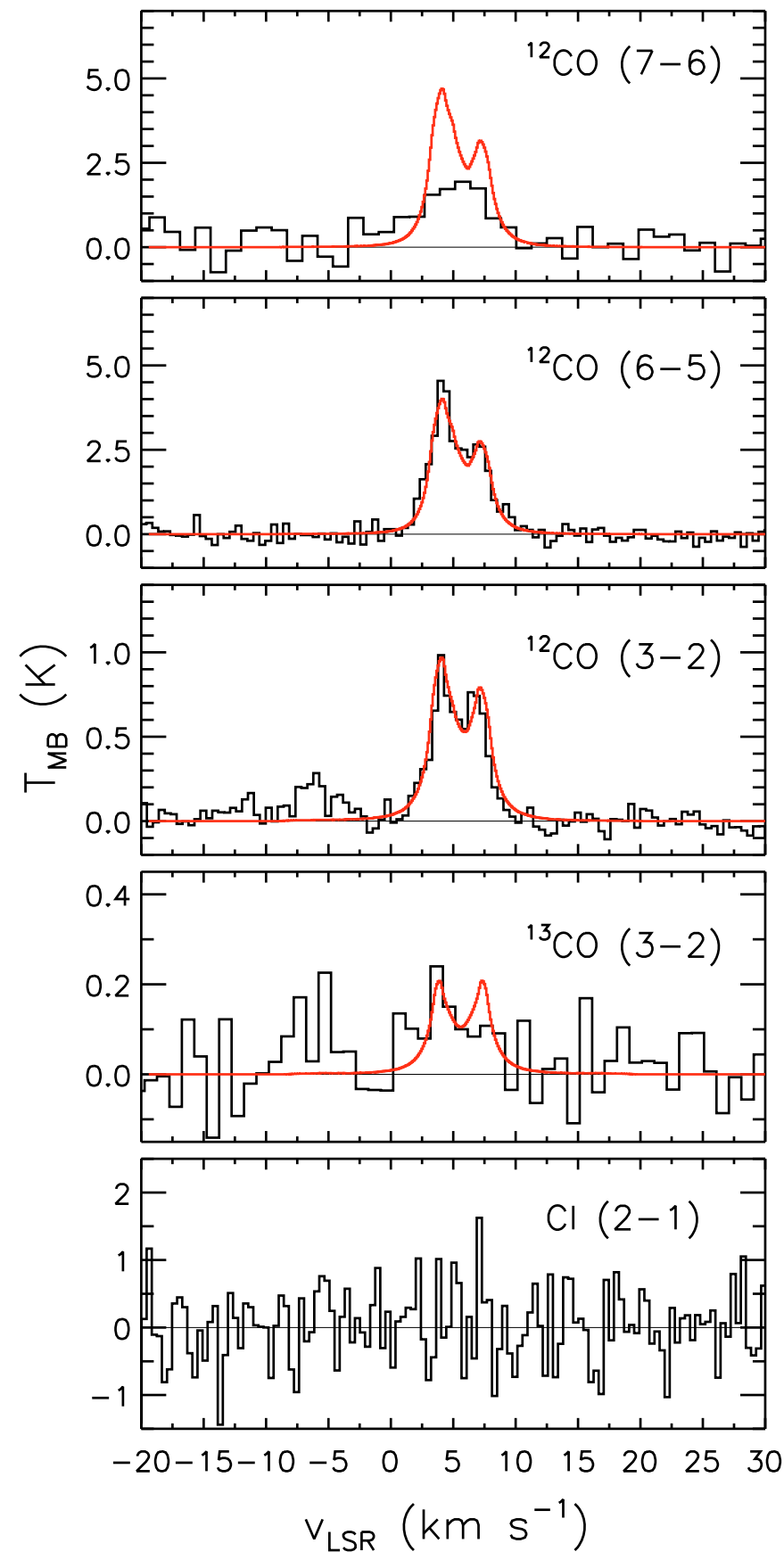

Fig. 1. From top to bottom: spectra of the ${ }^{12} \mathrm{CO} J=7-6, J=6-5, J=$ $3-2,{ }^{13} \mathrm{CO} J=3-2$ and $\mathrm{CI} J=2-1$ lines observed towards HD 100546 shown with black lines plotted as a histogram. The ${ }^{12} \mathrm{CO}$ and ${ }^{13} \mathrm{CO}$ spectra computed from our models of the line emitting layers of the disc (see Sects. 3.2.1 and 3.2.2) are shown with continuous red lines. At $100 \mathrm{AU}$ from the star, our vertically isothermal models of the ${ }^{12} \mathrm{CO}$ line emission have temperatures $T_{100}=60-70 \mathrm{~K}$, while a lower temperature $T_{100}=$ $30 \mathrm{~K}$ is used for the ${ }^{13} \mathrm{CO}$ line.

transitions at $7-20 \sigma$ in terms of the integrated intensity. The ${ }^{13} \mathrm{CO} J=3-2$ line is marginally detected at $2 \sigma$, and the $[\mathrm{C} \mathrm{I}] J=$ $2-1$ line is not detected. Figure 1 shows the observed spectra, corrected for the beam efficiency and re-binned to a lower spectral resolution. A first-degree polynomial fit, except for the $0-10 \mathrm{~km} \mathrm{~s}^{-1}$ range in which the lines are emitted, is subtracted from the full spectral range for each data set. The ${ }^{12} \mathrm{CO} J=3-2$ and $J=6-5$ lines are detected at the highest signal-to-noise ratio
Table 1. Integrated intensities of the observed spectral lines.

\begin{tabular}{ccc}
\hline \hline Spectral Line & $\begin{array}{c}I^{a} \\
\left(\mathrm{~K} \mathrm{~km} \mathrm{~s}^{-1}\right)\end{array}$ & $\begin{array}{c}F W H M \\
\left(\mathrm{~km} \mathrm{~s}^{-1}\right)\end{array}$ \\
\hline${ }^{12} \mathrm{CO} J=7-6$ & $12.9 \pm 1.9$ & 4.2 \\
${ }^{12} \mathrm{CO} J=6-5$ & $17.7 \pm 0.9$ & 4.2 \\
${ }^{12} \mathrm{CO} J=3-2$ & $4.0 \pm 0.6$ & 4.0 \\
${ }^{13} \mathrm{CO} J=3-2$ & $1.3 \pm 0.6^{b}$ & $(\ldots)$ \\
$\mathrm{CI} J=2-1$ & $<1.0^{c}$ & $(\ldots)$ \\
\hline
\end{tabular}

Notes. ${ }^{(a)}$ The line intensity $I=\int T_{\mathrm{MB}} \mathrm{d} V$ is integrated over the velocity range $0-10 \mathrm{~km} \mathrm{~s}^{-1}$; ${ }^{(b)}$ marginal $2 \sigma$ detection; ${ }^{(c)}$ upper limit given by the $2 \sigma$ value.

and show a double-peaked profile characteristic of disc rotation. The intensities integrated over the velocity range $0-10 \mathrm{~km} \mathrm{~s}^{-1}$, over which line emission is detected, are listed in Table 1, together with the full width at half-maximum $(F W H M)$ of the lines with sufficiently well-defined profiles.

In addition to the observations towards the source, the $\mathrm{CHAMP}^{+}$array provides measurements at nearby offsets. This setup provides an excellent way to discern the emission from the disc, with an estimated size of at least $400 \mathrm{AU}$ in radius from the surrounding material known to be present further away from the star (Henning et al. 1998; Grady et al. 2001; Augereau et al. 2001). The $400 \mathrm{AU}$ lower limit to the size of the gas disc is set by the scattered light observations (Augereau et al. 2001 ) is close to the spatial resolution of our data. The central pixel of our $\mathrm{CHAMP}^{+}$data probes the ${ }^{12} \mathrm{CO} J=6-5$ line emission from the region of $9^{\prime \prime}$ that is centered on the position of the star (460 AU radius), while the surrounding pixels probe the more distant regions (at a distance 1000-2000 AU from the star). Similarly, at the frequency of the ${ }^{12} \mathrm{CO} J=$ 7-6 line, a small 7". 7-sized region (400 AU radius) around the star is probed with the central pixel, whereas the surrounding pixels probe the regions that are located further away. Table 2 provides an overview of the pixel positions and the corresponding fluxes integrated over a velocity range from 0 to $10 \mathrm{~km} \mathrm{~s}^{-1}$, over which the ${ }^{12} \mathrm{CO}$ lines are firmly detected in the central pixel. Compared to the on-source fluxes, these measurements show that the ${ }^{12} \mathrm{CO}$ emission from the surrounding material is about 20 times weaker than from the region within $400 \mathrm{AU}$ from the star. The ${ }^{12} \mathrm{CO} J=3-2$ line was observed with a single pointing, and the beam of $18^{\prime \prime} .1$ is large enough to include any emission from regions beyond 400 AU. However, the strong resemblance in the line profile suggests that both low- $J$ and high- $J$ lines arise from the disc and that any contribution to the line emission by an extended low-temperature and low-velocity component is negligible.

Our data testify to the presence of warm molecular gas at distances less than roughly 400-500 AU from the star, corresponding to the smallest beam sizes in our observations ( 7.7 and $\left.9^{\prime \prime}\right)$. We obtain the following integrated intensity ratios, corrected for beam dilution (scaled to the same beam): ${ }^{12} \mathrm{CO}$ lines $(6-5) /(3-2)=1.1 \pm 0.6$ and $\left({ }^{12} \mathrm{CO} 3-2\right) /\left({ }^{13} \mathrm{CO} 3-2\right) \geq 2.8 \pm 2.2$. The errors include both the rms and flux calibration uncertainty.

There is a clear asymmetry in the profile of the ${ }^{12} \mathrm{CO} J=$ 3-2 and $J=6-5$ lines, observed at a high signal-to-noise ratio and characterised by a $20-40 \%$ difference in the line intensity between the two peaks. This asymmetry is certain, given that the spectra shown in Fig. 1 have been smoothed to a lower spectral resolution and that each spectral bin contains an average of several measurements. 
A\&A 519, A110 (2010)

Table 2. The ${ }^{12} \mathrm{CO} J=6-5$ and $J=7-6$ integrated line intensities at offset positions.

\begin{tabular}{cccccc}
\hline \hline $\begin{array}{c}{ }_{\text {RA offset }} \\
\left({ }^{\prime \prime}\right)\end{array}$ & $\begin{array}{c}\mathrm{CO} J=6-5 \\
\mathrm{Dec} \text { offset } \\
\left({ }^{\prime \prime}\right)\end{array}$ & $\begin{array}{c}I_{\mathrm{CO}(6-5)} \\
\left(\mathrm{K} \mathrm{km} \mathrm{s}^{-1}\right)\end{array}$ & $\begin{array}{c}{ }^{12} \mathrm{CO} J=7-6 \\
\left({ }^{\prime \prime}\right)\end{array}$ & $\begin{array}{c}\mathrm{Dec} \text { offset } \\
\left({ }^{\prime \prime}\right)\end{array}$ & $\begin{array}{c}I_{\mathrm{CO}(7-6)} \\
\left(\mathrm{K} \mathrm{km} \mathrm{s}^{-1}\right)\end{array}$ \\
\hline-9.8 & -17.0 & $0.4 \pm 0.2$ & +10.2 & -17.0 & $<2.0$ \\
+8.8 & -18.0 & $0.5 \pm 0.2$ & +20.2 & -1.4 & $<2.0$ \\
-18.9 & -0.2 & $<0.2$ & -9.0 & -16.5 & $<2.0$ \\
+19.4 & -1.2 & $<0.2$ & +11.0 & +16.2 & $<2.0$ \\
-9.3 & +16.6 & $<0.2$ & -18.8 & +0.2 & $<2.0$ \\
+10.0 & +16.6 & $<0.2$ & -9.1 & +16.9 & $<2.0$ \\
\hline
\end{tabular}

Notes. The line intensities $I=\int T_{\mathrm{MB}} \mathrm{d} V$ are integrated over a velocity range $0-10 \mathrm{~km} \mathrm{~s}^{-1}$. The upper limits are given by the $2 \sigma$ value.

In some sources, asymmetries like this are explained through confusion with foreground cloud emission, as seen in ${ }^{12} \mathrm{CO}$ lines towards IM Lup (van Kempen et al. 2007) and DL Tau (Simon et al. 2000). However, in the off-source pixels of the CHAMP array, we see no significant extended emission. While a slight pointing offset in the $6-5$ line observations towards a symmetric source may result in a line asymmetry, the persistance of line asymmetry in our $188^{\prime \prime} 1$ resolution observations of the 3-2 line indicates that the asymmetry is caused by the source properties. Furthermore, the two observations were taken years apart, so a systematic offset in both observations is highly unlikely. We therefore exclude pointing offset as a cause of the observed line asymmetry. If one side of the disc extends slightly further out than the other (e.g., on the SE side), the increase in disc surface on that side will contribute to the line flux on one side of the spectral line, and result in line asymmetry. This may be a plausible explanation for the 3-2 line, but not the observed 6-5 line with a $9^{\prime \prime}$ beam size, unless the asymmetry is within $450 \mathrm{AU}$. Such an asymmetry may affect the scattered light images of HD 100546, with the disc extending further to the SE than to the NW of the star. However, this is not seen in the observations of Augereau et al. (2001). Furthermore, the additional emission from this region would dominate the frequencies closer to the line centre and not the blue-shifted peak.

Asymmetry is seen in ${ }^{12} \mathrm{CO}$ lines from sources with a pronounced structural disc asymmetry, like HD 141569 (Dent et al. 2005). Another possibility is a temperature asymmetry in the disc. In the following section, we analyse and model the observed lines and investigate these scenarios.

\section{Discussion}

\subsection{Line ratios as temperature and density diagnostics}

\subsection{1. ${ }^{12} \mathrm{CO} 3-2 /{ }^{13} \mathrm{CO} 3-2$ line ratio and disc density}

The submillimetre ${ }^{12} \mathrm{CO}$ line emission from circumstellar discs is generally optically thick and arises from warm upper disc layers. These lines are particularly sensitive to the temperature of these layers, and therefore to the stellar and external illumination of the disc surface. Comparison to isotopologue line emission is often used to assess the optical thickness. If the ${ }^{13} \mathrm{CO} 3-2$ emission is treated as a detection, we obtain the line ratio of $\left({ }^{12} \mathrm{CO} 3-2\right) /\left({ }^{13} \mathrm{CO} 3-2\right)=2.8 \pm 2.2$. We make a rough estimate of the column density needed to reproduce the derived ratio using the RADEX online tool ${ }^{1}$ (van der Tak et al. 2007). For kinetic temperatures $20-40 \mathrm{~K}, \mathrm{H}_{2}$ densities $10^{6}-10^{8} \mathrm{~cm}^{-3}$,

${ }^{1}$ http://www.strw.leidenuniv.nl/ moldata/radex.html and an isotopic ratio $\left[{ }^{12} \mathrm{C}\right] /\left[{ }^{13} \mathrm{C}\right]=77$ (Wilson \& Rood 1994), the $\left({ }^{12} \mathrm{CO} 3-2\right) /\left({ }^{13} \mathrm{CO} 3-2\right)$ ratio derived above indicates the ${ }^{12} \mathrm{CO}$ column density of roughly $10^{18} \mathrm{~cm}^{-2}$, and excludes lower column densities. Using a standard ${ }^{12} \mathrm{CO}$ abundance of $10^{-4}$ with respect to $\mathrm{H}_{2}$, this translates to the gas column density of more than $10^{22} \mathrm{~cm}^{-2}$.

\subsection{2. ${ }^{12} \mathrm{CO} 6-5 /{ }^{12} \mathrm{CO} 3-2$ line ratio and disc temperature}

We compared the line ratio $\left({ }^{12} \mathrm{CO} 6-5\right) /\left({ }^{12} \mathrm{CO} 3-2\right)=1.1 \pm 0.6$ to the calculations done using RADEX, and assumed that these two lines are dominated by the same region in the disc. This assumption is generally true up to 200-300 AU from the star (see calculations done in van Zadelhoff et al. 2001b). For $\mathrm{H}_{2}$ densities of $10^{6}-10^{8} \mathrm{~cm}^{-3}$, and ${ }^{12} \mathrm{CO}$ column densities of $10^{17}-10^{19} \mathrm{~cm}^{-2}$, the $\left({ }^{12} \mathrm{CO} 6-5\right) /\left({ }^{12} \mathrm{CO} 3-2\right)$ line ratio $\approx 1.1$ is reproduced at temperatures of $60-100 \mathrm{~K}$. If the disc is large and sufficiently cold, the assumption that the two lines arise from the same region is not necessarily true, as the 3-2 line may be dominated by colder material at radii larger than the $6-5$ line. It is therefore uncertain whether the line ratio reflects the difference in the temperature between the two vertical layers or in the temperature of the radial regions emitting the lines. We explore this further in Sect. 3.2.1 using more sophisticated radiative transfer tools and parametric models of disc structure.

The observed CO 6-5/3-2 integrated intensity ratio of $1.1 \pm$ 0.6 (observations scaled by the beam size) is higher than the ratios close to 0.5 found for the discs around the T Tauri stars LkCa 15 and TW Hya by van Zadelhoff et al. (2001a) and Qi et al. (2006). The TW Hya 6-5/3-2 ratio has been interpreted as proof that the gas temperature is higher than that of the dust in the surface layers where gas and dust are not thermally coupled. Both UV radiation and X-rays have been invoked to provide the additional gas heating (Jonkheid et al. 2004; Kamp \& Dullemond 2004; Glassgold et al. 2004; Nomura \& Millar 2005; Gorti \& Hollenbach 2008), although the X-rays can be neglected for Herbig Ae stars (Kamp et al. 2008).

The higher ratio found for HD 100546 implies higher gas temperatures than for the aforementioned discs around T Tauri stars. This is expected based on models where most of the gas heating comes from UV radiation from the central star, since the cooler stars have less UV radiation (e.g., Woitke et al. 2009). The gas temperature is also strongly affected by the PAH abundance in the disc. PAH features are prominent in the HD 100546 mid-infrared spectrum (e.g., Malfait et al. 1998), and have been shown to be spatially extended across the disc (Geers et al. 2007). In contrast, neither of the two T Tauri discs for which the 
${ }^{12} \mathrm{CO} J=6-5$ line has been detected show PAH emission, implying typical PAH abundances that are a factor of 10-100 lower (Geers et al. 2006).

For the specific case of Herbig Ae discs, Jonkheid et al. (2007) have computed the gas heating and chemistry, as well as the resulting $\mathrm{CO} 6-5$ and 3-2 line emission, starting from a set of dust disc models developed by Dullemond \& Dominik (2005). Models with decreasing disc mass from $10^{-1}$ to $10^{-4} M_{\odot}$ and decreasing dust/gas ratios from $10^{-2}$ to $10^{-6}$ (simulating grain growth and settling) were investigated. The UV radiation field was taken to be that of a B9.5 star (see Fig. 3 of Jonkheid et al. 2006), very close to what is expected for HD 100546. An accurate treatment of the shape of the UV field at short wavelengths, $<1100 \AA$, is very important for correct calculation of the $\mathrm{CO}$ photodissociation and atomic carbon photoionisation rates, since the UV intensity of a B9 star is orders of magnitude weaker in this wavelength range than the (scaled) standard interstellar radiation field. The PAH abundance is taken to follow the dust/gas ratio, with an abundance of $10^{-7}$ for a normal dust/gas ratio of 0.01 .

The ${ }^{12} \mathrm{CO} J=6-5 / 3-2$ integrated intensity ratios computed by Jonkheid et al. (2007) summed over the full extent of the disc model are remarkably close to unity for the entire range of disc parameters investigated, consistent with our observations. The absolute ${ }^{12} \mathrm{CO}$ and ${ }^{13} \mathrm{CO}$ intensities for model B2 (a disc with a mass of $0.01 M_{\odot}$ with a standard gas/dust ratio of 100 , appropriate for HD 100546) are also within $40 \%$ of the observed values when scaled to the same source distance and beam size, indicating good agreement between models and observations.

\subsection{Parametric disc models and origin of ${ }^{12} \mathrm{CO}$ line emission}

Submillimetre ${ }^{12} \mathrm{CO}$ line emission is analysed using two different modelling approaches in the literature. Disc physical models, like the irradiated accretion disc models of D'Alessio et al. (2005); Dullemond \& Dominik (2005), are especially suitable when the emission is spatially resolved. In this way, the threedimensional structure of the disc can be investigated, for example when transitions of different optical depths are observed (Panić et al. 2008). For spatially unresolved observations, like those presented here, simplistic models with a limited number of free parameters are more appropriate for deriving some basic constraints on disc properties based on the line spectrum (method employed in, e.g., Dutrey et al. 1994; Guilloteau \& Dutrey 1998; van Zadelhoff et al. 2001a; Dartois et al. 2003; Dent et al. 2005). Here we go a step further than in Sect. 3.1 in analysing the observations in the context of such models.

Density and temperature structure. Panić \& Hogerheijde (2009) show that simple power-law disc models, with a disc mass $M=0.01 M_{\odot}$, an $\mathrm{H}_{2}$ surface density $\Sigma \propto R^{-1}$, and temperature $T=T_{100} \mathrm{~K}(R / 100 \mathrm{AU})^{-q}$ with $T_{100}=60 \mathrm{~K}$ and $q=0.5$, are useful tools for the analysis of low $-J{ }^{12} \mathrm{CO}$ transitions from discs around gas-rich Herbig Ae stars. These models are vertically isothermal and have a vertical density structure in hydrostatic equilibrium. In the upper panels of Fig. 2 the temperature and surface density are shown of one of these models that has an outer radius of $400 \mathrm{AU}$. The ${ }^{12} \mathrm{CO}$ abundance with respect to $\mathrm{H}_{2}$ is assumed to be $10^{-4}$, constant throughout the disc. Their analysis uses the assumption that the low- $J{ }^{12} \mathrm{CO}$ lines arise from the upper disc layers (optically thick) and that their fluxes are dominated by the outermost disc regions. Therefore the spectral profiles of these lines are very sensitive to the extent of the emission, with strong and narrow lines indicating a large disc and weak and relatively wider double-peaked lines characteristic of small discs.

Spatial origin of the emission. The regions of the disc probed by the ${ }^{12} \mathrm{CO} J=6-5$ and $J=3-2$ lines are studied in van Zadelhoff et al. (2001b). Their Fig. 6 shows the locations of the surfaces of opacity $\tau=1$ for these lines in a cross-section of their disc model. At distances up to $150 \mathrm{AU}$ from the star, the two transitions trace similar depths into the discs, but at larger radii they trace different vertical layers. The low-excitation 3-2 line probes deeper and colder layers than the 6-5 line. To assess whether both 6-5 and 3-2 line fluxes are dominated by the same radial disc region and to identify this region, we used the calculations of synthetic 6-5 and 3-2 spectra from disc models as described in the previous paragraph. We used two different temperature profiles with $q=0.5$ and $q=0.2$. The radial temperature and surface density structure of the two models is shown in Fig. 2. In this exercise we use the face-on disc orientation for simplicity, and calculate the emerging ${ }^{12} \mathrm{CO} \mathrm{J}=$ 6-5 and $J=3-2$ line intensity along lines of sight at a number of different radial locations from the star. This is done using the molecular excitation and radiative transfer code RATRAN (Hogerheijde \& van der Tak 2000) and $\mathrm{CO}-\mathrm{H}_{2}$ collision rates from the Leiden Atomic and Molecular Database ${ }^{2}$ (LAMBDA; Schöier et al. 2005). We are primarily interested in knowing if there is a single radial region dominating the line flux more than other regions or the flux is distributed more evenly with the radius. Also, we investigate how the regions of strongest line flux spatially compare for the two ${ }^{12} \mathrm{CO}$ transitions. The contribution to the line flux from an annulus of a fixed width $\mathrm{d} R$ located at a distance $R$ from the star is given by $\mathrm{d} F(R)=2 \pi I(R) R \mathrm{~d} R$, hence $\mathrm{d} F(R) \propto I(R) R$. The relative contribution of the annuli to the total line flux arising from the model $\mathrm{d} F(R) / F_{\text {total }}$ is also proportional to $I(R) R$, allowing us to directly compare how much one radial region contributes to the line flux with respect to another region. Figure 2 shows $\mathrm{d} F(R)$ for the ${ }^{12} \mathrm{CO} J=6-5$ and $J=$ $3-2$ in case $q=0.5$ (upper panels) and $q=0.2$ (lower panels). For ease of comparison between the two lines, the ${ }^{12} \mathrm{CO} J=3-2$ and $J=6-5$ line fluxes are plotted with respect to their maximum value $\mathrm{d} F_{\max }$. For both temperature profiles, the outermost disc region (200-400 AU) is very important, especially for the 3-2 line. For this line the strongest flux arises at the largest radii due to the efficient excitation at low temperatures found in the outer disc combined with the large surface area. This result is in line with our previous results in Panić \& Hogerheijde (2009) where the ${ }^{12} \mathrm{CO} J=3-2$ spectra are used as probes of disc sizes. While the 6-5 line is also sensitive to the outer disc regions, the upper panels of Fig. 2 show that our model assumptions at $50 \mathrm{AU}$ matter just as much as those at $400 \mathrm{AU}$. This is explained by the excitation energy of the ${ }^{12} \mathrm{CO} J=6-5$ line, what is somewhat high for the 30-40 K temperatures found in the outer disc in the model with a steeper temperature profile $q=0.5$. The flatter temperature distribution with $T>40 \mathrm{~K}$ in the outer disc allows the higher energy levels of ${ }^{12} \mathrm{CO}$ to be efficiently populated, and in this case the outermost disc regions are clearly more important than 50-100 AU, merely because of the increase in the surface area with radius. Understanding the diagnostic potential of the high- $J{ }^{12} \mathrm{CO}$ lines in discs is already useful for the analysis of spatially unresolved APEX observations (see Sect. 3.2.1), but also shows that the future sub-arcsecond observations of high- $J$ ${ }^{12} \mathrm{CO}$ lines in discs with ALMA could be used to probe the radial temperature structure in discs.

2 http://www.strw.leidenuniv.nl/ moldata/ 

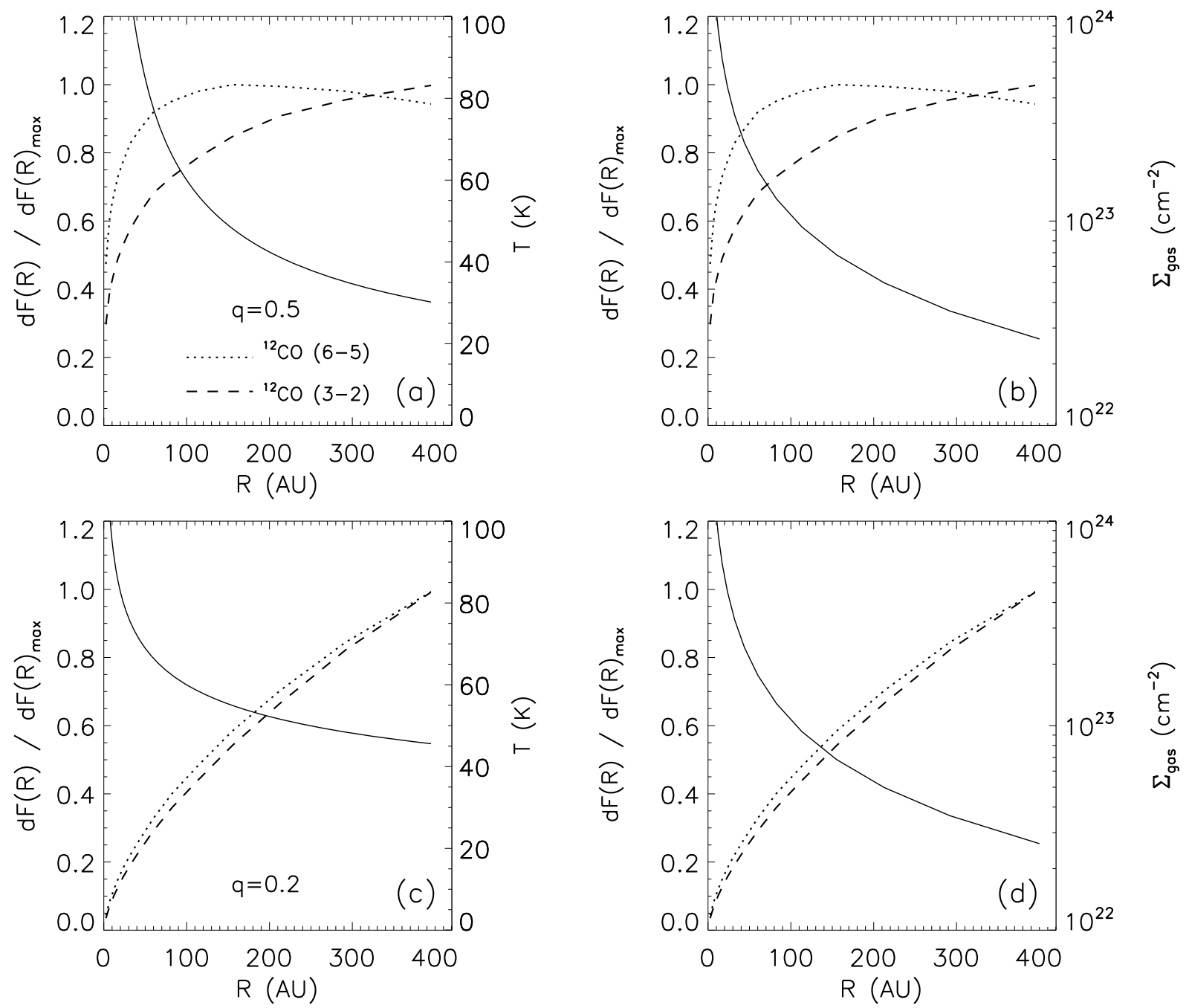

Fig. 2. a): radial temperature structure of a disc model from Panić \& Hogerheijde (2009), with $R_{\text {out }}=400 \mathrm{AU}, T_{100}=60 \mathrm{~K}$ and $q=0.5$ is shown with the black solid line. The radial dependence of the line flux $\mathrm{d} F(R) / \mathrm{d} F(R)_{\max }$ for the ${ }^{12} \mathrm{CO} 3-2$ line and $6-5$ line arising from this model is overplotted using the dashed and the dotted lines, respectively. b): the surface density of the same model is shown with the black full line, and line fluxes as in a). c) and d): same as in a) and b), for the model with a shallower temperature profile, $q=0.2$ and its corresponding line fluxes as a function of radius.

Disc mass. Our initial choice of parameters, described above, roughly matches the observed ${ }^{12} \mathrm{CO} J=6-5$ and $J=3-2$ line fluxes, confirming the outer radius of $400 \mathrm{AU}$ seen in scattered light observations. We performed test calculations for a lower value of the disc mass of $10^{-3} M_{\odot}$, i.e., ten times lower densities. The modelled line fluxes are found not to vary significantly with respect to the noise levels of our observations. Thus, in the disc mass range of $10^{-3}-10^{-2} M_{\odot}$, values found typically towards circumstellar discs and temperatures similar to those assumed here, the observed ${ }^{12} \mathrm{CO} J=6-5$ and $J=3-2$ lines are insensitive to disc mass. If we decrease the disc mass to allow the ${ }^{12} \mathrm{CO}$ line emission in our models to be sensitive to the density variations in the midplane (i.e., emission dominated by the midplane), the disc midplane temperatures $(\leq 20 \mathrm{~K}$ in the outer disc) would be the only physically appropriate assumption for the temperature in the "low-mass" model. While the asymmetry in the line profile could be reproduced in this way, the resulting lines would be much weaker than observed. Given these considerations, we assume the two lines to be optically thick in the disc around HD 100546 and fix the disc mass to the initial value of $10^{-2} M_{\odot}$. Our model is consistent with the thermal continuum emission of dust observed toward HD 100546 by Henning et al. (1998) and Wilner et al. (2003). In the further analysis, we fit the exact line shapes and intensities, with a particular focus on the line asymmetry seen in both $J=6-5$ and $J=3-2$ spectra.

\subsection{1. ${ }^{12} \mathrm{CO} J=6-5$ and $J=3-2$ line fit}

The characteristic temperatures and masses derived in Sect. 3.1 for the disc around HD 100546 are similar to the models used in Panić \& Hogerheijde (2009). The modelling of the ${ }^{12}$ CO 3-2 line of HD 100546 in Panić \& Hogerheijde (2009) yielded very rough estimates of the outer radius of $300 \mathrm{AU}$ and inclination of $35^{\circ}$, consistent with the $400 \mathrm{AU}$ and $50^{\circ}$ from the scattered light. We use their models to fit the ${ }^{12} \mathrm{CO}$ spectra, with $T_{100}$ as free parameter and varying $q$ from its initial value of 0.5 where necessary. We fix the outer radius and inclination to the observationally constrained values $400 \mathrm{AU}$ and $50^{\circ}$ (see Augereau et al. 2001, and the discussion of disc size in Sect. 2). We use the Keplerian velocity field around a $2.5 M_{\odot}$ star. The dependence of the spectral profile on the stellar mass in the range 2.0-3.0 $M_{\odot}$ does not affect our fit significantly. 
The inner radius is assumed to be $0.6 \mathrm{AU}$, close to the dust sublimation radius. Although an inner hole of $13 \mathrm{AU}$ is found in HD 100546, its presence would not affect our results because the molecular lines observed are dominated by disc regions far beyond the inner tens of $\mathrm{AU}$. Because the observed ${ }^{12} \mathrm{CO}$ lines trace warm molecular material $(>20 \mathrm{~K})$ and are insensitive to the colder regions deeper in the disc, it is reasonable to neglect the ${ }^{12} \mathrm{CO}$ freeze-out.

As above, the line emission is calculated using RATRAN. The synthetic images from our disc models are convolved with the beam sizes of the corresponding observations, and the spectra are extracted towards the centre of the image. Where temperature asymmetry is modelled (see 3.2.1), pairs of synthetic images with different $T_{100}$ parameter were first combined - each contributing to a given side of the disc with respect to the semimajor axis. Following this, the combined image is convolved and spectra extracted. Although negligible for the molecular line transfer, dust continuum emission is included in the calculation and subtracted from the synthetic image cubes.

In view of the result that the line emission appears to be insensitive to the density, the observed line asymmetry cannot be caused by a density asymmetry in the disc, but rather reflects an asymmetry in the disc temperature structure. To model such an asymmetry, we use the fact that the emission at the two peaks in the line profile is dominated by the two sides of the disc with respect to the minor axis. We assume that one side of the disc is colder than the other. This may stem from a combined effect of an asymmetry in disc illumination and geometry. We use different $T_{100}$ parameters for the two sides of the disc and explore different radial temperature distributions with $T \propto R^{-q}, q=0-0.5$.

The best fit to the ${ }^{12} \mathrm{CO} J=3-2$ spectrum, seen in Fig. 1, is obtained by assuming the radial temperature structure with $q=0.5$, as in Panić \& Hogerheijde (2009), and $T_{100}$ of 60 and $70 \mathrm{~K}$ for the two disc sides, respectively. The line shape is consistent with the assumed Keplerian velocity field and disc inclination. The temperatures of $60-70 \mathrm{~K}$ compare well to the theoretical predictions of temperature in the regions where these lines saturate in discs (see Fig. 6 in van Zadelhoff et al. 2001a). They show that at $100 \mathrm{AU}$ from the star, the $J=6-5$ and $J=$ 3-2 lines trace the same disc layer. Therefore we assume that the ${ }^{12} \mathrm{CO} J=6-5$ line is emitted at $T_{100}$ of 60 and $70 \mathrm{~K}$, as found for the 3-2 line. We find the best fit to the ${ }^{12} \mathrm{CO} J=6-5$ spectrum, shown in Fig. 1, assuming $T=60 \mathrm{~K} \times(R / 100 \mathrm{AU})^{-0.3}$ on one side of the disc and $T=70 \mathrm{~K} \times(R / 100 \mathrm{AU})^{-0.2}$ on the other. Thus, the radial temperature distribution in the $6-5$ line emitting layer is flatter than in the 3-2 layer, consistent with the result of van Zadelhoff et al. (2001b) for the outer disc regions where the 6-5 line traces warmer material higher in the disc surface than the 3-2 line.

Therefore our ${ }^{12} \mathrm{CO}$ line observations, although spatially unresolved, provide some information on the temperature radial distribution in the 6-5 and 3-2 line emitting layers and disc temperature asymmetry. It is important to note that slightly different sets of temperature values and slopes than those found here may provide equally good fits to these observations. However, any such model requires higher outer disc temperatures to fit the 6-5 line than required for the 3-2 line, clearly showing that the two lines arise from different vertical layers in the outermost disc regions.

The difference in temperature between the two sides of the disc may be explained by a warped inner disc, as illustrated in Fig. 3, with the elevated side of the inner disc intercepting a fraction of stellar light that would otherwise reach the outer disc, while the opposite side is slightly more illuminated, with the

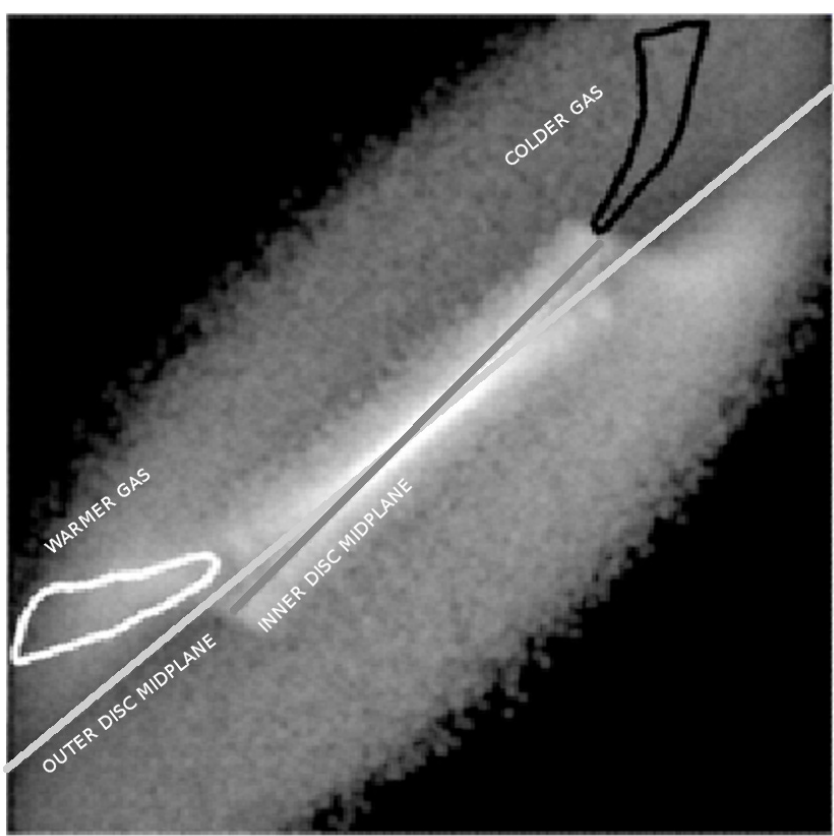

Fig. 3. Illustration of a warped disc structure, in an edge-on projection. Straight light and dark grey lines show the plane of the disc and of the inner warped disc component, respectively. The white curve shows the surface region with a higher temperature, heated well by the star, and the black curve shows the cooler region partly obscured by the warp. The image of the disc with a $400 \mathrm{AU}$ radius is adapted from Quillen (2006), where a detailed warped disc model is presented.

inner part of the disc shifted downwards. The possibility of an inner warp is suggested in Quillen (2006) for HD 100546, with an inner component extending up to $200 \mathrm{AU}$ inclined by $\approx 15^{\circ}$ with respect to the outer component extending beyond that radius.

The temperature asymmetry is also possible if the disc has a different thickness on the two sides. This may happen in a disc with dust settling underway, if a planet or another body embedded in the disc stirs the dust back up. In this case, the "stirred" part of the disc intercepts more stellar light and becomes somewhat warmer. A companion body in HD 100546 is suggested in the literature, to explain the observed inner hole, gas kinematics, and spiral arms (Quillen et al. 2005; Acke \& van den Ancker 2006b).

\subsection{2. ${ }^{13} \mathrm{CO} J=3-2$ and ${ }^{12} \mathrm{CO} J=7-6$ line fit}

With the low signal to noise in our ${ }^{13} \mathrm{CO} J=3-2$ spectrum (marginal detection at $2 \sigma$ ), it is not possible to assess the spectral line shape. The disc temperatures used to fit the ${ }^{12} \mathrm{CO}$ lines in Sect. 3.2.1 overestimate the ${ }^{13} \mathrm{CO} J=3-2$ line intensity. A lower temperature of the ${ }^{13} \mathrm{CO} J=3-2$ emitting layers with respect to the ${ }^{12} \mathrm{CO} J=3-2$ (as discussed in Sect. 3.2.2), a higher $\left[{ }^{12} \mathrm{C}\right] /\left[{ }^{13} \mathrm{C}\right]$ isotopic ratio than the standard interstellar medium value of 77 , or an overall decrease in $\mathrm{CO}$ abundance can explain our marginal detection of the ${ }^{13} \mathrm{CO}$ line. Freeze-out and/or selective photodissociation processes in discs are known to affect the CO abundance and isotopic ratios. Detailed modelling of spatially resolved submillimetre line observations of ${ }^{12} \mathrm{CO}$ and isotopologues would allow us to constrain the disc structure and the amount of molecular gas better, shedding light on these processes (e.g., Panić et al. 2008). Thereby, there are a number of uncertainties involved in the interpretation of our ${ }^{13} \mathrm{CO} J=$ 3-2 data. For these reasons we limit the analysis to a comparison 
of our marginal detection to one simple model. A lower temperature of the ${ }^{13} \mathrm{CO}$ emitting layers is expected because of a different optical depth of ${ }^{13} \mathrm{CO}$ lines with respect to the ${ }^{12} \mathrm{CO}$. In Fig. 1 we compare our data to model calculations done assuming low temperatures, $T_{100}=30 \mathrm{~K}$ and $q=0.5$, an isotopic ratio $\left[{ }^{12} \mathrm{C}\right] /\left[{ }^{13} \mathrm{C}\right]=77$, and all remaining model parameters as in the simple axially symmetric power-law model shown in Fig. 2 and described in Sect. 3.2.1. Considering that the ${ }^{13} \mathrm{CO}$ line emission probes colder and deeper disc layers, it is likely that temperature asymmetry derived for the upper layers from ${ }^{12} \mathrm{CO}$ lines is negligible for ${ }^{13} \mathrm{CO}$. High-sensitivity observations of ${ }^{13} \mathrm{CO}$ or $\mathrm{C}^{18} \mathrm{O}$ submillimetre lines in the future may reveal symmetric line profiles.

The ${ }^{12} \mathrm{CO} J=7-6$ and $J=6-5$ transitions arise from energy levels at $E_{\mathrm{k}}$ of $155 \mathrm{~K}$ and $116 \mathrm{~K}$, respectively, and are expected to trace disc layers that are roughly the same. Figure 1 shows how our best-fit model of the 6-5 line compares to the observed 7-6 line. The observed line flux is about $50 \%$ lower than the model prediction. This may be due to a slight mispointing of the telescope, as the size of the source (800 AU in diameter) is close to the $7^{\prime \prime} .7$ beam size at this frequency. The noise levels of these observations are notably higher than in the 6-5 line observations, and spectral profile not well defined. For these reasons, we do not fit the 7-6 line further but use the more reliable 6-5 line fit results in our analysis.

\subsection{Implications of the $[C I] J=2-1$ non-detection}

Figure 1 includes the high-quality spectrum around the [C I] $J=$ 2-1 line at $809.344 \mathrm{GHz}$. No significant feature is detected down to $0.3 \mathrm{~K} \mathrm{rms}$ in a $0.27 \mathrm{~km} \mathrm{~s}^{-1}$ velocity bin, implying a $2 \sigma$ limit on the integrated intensity of $\approx 1.0 \mathrm{~K} \mathrm{~km} \mathrm{~s}^{-1}$ over the range $0-10 \mathrm{~km} \mathrm{~s}^{-1}$ (same width as for the detected ${ }^{12} \mathrm{CO}$ lines). Model B2 of Jonkheid et al. (2007) predicts integrated [C I] intensities, scaled to the distance of HD 100546, around $15-20 \mathrm{~K} \mathrm{~km} \mathrm{~s}^{-1}$, whereas the model intensities are even higher in discs with significant grain growth and settling (BL model series in the aforementioned paper). Thus, while the CO data appear entirely consistent with their sophisticated UVheated disc atmosphere models, the [C I] data are clearly discrepant by an order of magnitude.

One possible solution could be that the radiation field contains more carbon ionising photons than assumed here, shifting the chemical balance from neutral to ionised atomic carbon. The predicted [C II] line intensities for the model discs of Jonkheid et al. (2007) are very low, $<0.1 \mathrm{~K} \mathrm{~km} \mathrm{~s}^{-1}$, whereas they are more than an order of magnitude higher for discs around $\mathrm{T}$ Tauri stars with excess UV emission (Jonkheid et al. 2004), consistent with an efficient carbon ionisation around Herbig Ae stars. Such excess of UV emission could come from the disc-star accretion boundary layer. Indeed, HD 100546 is observed to undergo significant accretion, in spite of the known (dust) gap in the inner disc (Vieira et al. 1999). Also, the FUSE UV spectrum of HD 100546 shows significant flux, both in the continuum and in lines such as $\mathrm{O} \mathrm{VI}$, in the wavelength range where atomic carbon can be ionised (Lecavelier des Etangs et al. 2003, and G. Herczeg, priv. comm.). The continuum emission should also photodissociate $\mathrm{CO}$, but not the stellar UV lines unless they overlap with the discrete $\mathrm{CO}$ photodissociating transitions. Also, because $\mathrm{CO}$ is self-shielding and the lines are optically thick, the $\mathrm{CO}$ emission may be less affected than that of atomic carbon by any extra UV. However, detailed chemical modelling is required to test this hypothesis.
[C II] $158 \mu \mathrm{m}$ emission has been detected by Malfait et al. (1998) with ISO-LWS and most recently with the PACS instrument on the Herschel Space Observatory by Sturm et al. (2010). The ISO fluxes are up to an order of magnitude larger than the model fluxes by Jonkheid et al. (2007), but a significant fraction of the emission observed in the large ISO beam may be caused by general Galactic background emission. More detailed modelling is needed to pin down the origin of the absence of the [C I] $809 \mu \mathrm{m}$ line and check whether the observed [C II] flux is consistent with enhanced UV radiation. Future observations of the [C I] $J=1-0$ line at $492.161 \mathrm{GHz}\left({ }^{3} \mathrm{P}_{1}-{ }^{3} \mathrm{P}_{0}\right)$, which is generally brighter than the [C I] $J=2-1$ line, may provide a stronger constraint on the amount of neutral atomic carbon in the disc. Observations of the [C II] line with Herschel-HIFI can directly resolve the line profile and determine whether this line indeed comes from the disc. Overall, the [C I] flux and [C I]/[C II] line ratio could become interesting diagnostics of the UV radiation field to which the disc is exposed.

\section{Conclusions}

We summarise our conclusions as follows.

- We present evidence of warm molecular gas associated with the disc around HD 100546, in the regions within $400 \mathrm{AU}$ from the star, successfully separated from more extended material in our $\mathrm{CHAMP}^{+}$observations.

- The gas kinematics are consistent with Keplerian rotation around an $2.5 M_{\odot}$ star of a disc with a $400 \mathrm{AU}$ radius, viewed at an inclination of $50^{\circ}$ from face-on.

- The ${ }^{12} \mathrm{CO}(6-5) /(3-2)$ line ratio of $1.1 \pm 0.6$ is higher than measured towards discs around $\mathrm{T}$ Tauri stars, likely due to a more efficient gas heating of the disc containing PAHs by the stronger UV radiation from the B9 star.

- Our data testify to the significant molecular gas reservoir in the disc, consistent with the total disc masses of more than $10^{-3} M_{\odot}$. We exclude the possibility of a low-density disc and optically thin ${ }^{12} \mathrm{CO}$ emission.

- Line asymmetry seen in ${ }^{12} \mathrm{CO} J=6-5$ and $J=3-2$ lines is explained by a temperature asymmetry, with one side of the disc slightly colder than the other, possibly from partial obscuration of one side by a warped inner disc or a high disc rim. We exclude radial asymmetry, midplane density asymmetry, and mispointing as possible causes

- Our modelling shows that, owing to the efficient heating of the disc gas by the star, both low- $J$ and high- $J{ }^{12} \mathrm{CO}$ lines are dominated by the outermost regions of the disc, though slightly different vertical disc layers with $\Delta T=15-20 \mathrm{~K}$.

- We find that in "colder" discs where temperatures of the emitting regions are close to $20-30 \mathrm{~K}$ in the outer disc colder Herbig Ae discs and especially $\mathrm{T}$ Tauri discs - the high- $J$ lines probe a larger extent of the disc, starting from as little as $50 \mathrm{AU}$.

- The non-detection of [C I] $J=2-1$ line may indicate the presence of more carbon-ionising photons than assumed in the B9 model atmosphere.

Future observations with ALMA will allow us to characterise the disc around HD 100546 and spatially resolve its kinematics and structure. In particular, these observations will allow a detailed comparison between the spatial distribution of the gas traced by the rotational transitions of ${ }^{12} \mathrm{CO}$ and its isotopologues, and the dust traced with the millimetre continuum emission. Herschel far-infrared data can probe even higher $J{ }^{12} \mathrm{CO}$ transitions, as well as [O I] and [C II] lines originating from the disc 
surface. Because it is a bright, isolated source suspected of harbouring a planet, the disc around HD 100546 is one of the prime targets to probe disc structure in the early planet-building phases.

Acknowledgements. The research of O.P. and M.R.H. is supported through a VIDI grant from The Netherlands Organisation for Scientific Research (NWO). We thank L. Kristensen for advice regarding the APEX data reduction, and C. M. Wright for useful discussions. We thank the APEX staff for assistance during the observations. Construction of $\mathrm{CHAMP}^{+}$is a collaboration between the Max-Planck-Institut für Radioastronomie Bonn, The Netherlands Institute for Space Research (SRON), The Netherlands Research School for Astronomy (NOVA), and the Kavli Institute of Nanoscience at Delft University of Technology, with support from NWO grant 60006331010.

\section{References}

Acke, B., \& van den Ancker, M. E. 2006a, A\&A, 457, 171

Acke, B., \& van den Ancker, M. E. 2006b, A\&A, 449, 267

Ardila, D. R., Golimowski, D. A., Krist, J. E., et al. 2007, ApJ, 665, 512

Augereau, J. C., Lagrange, A. M., Mouillet, D., \& Ménard, F. 2001, A\&A, 365, 78

Beckwith, S., \& Sargent, A. 1987, in Circumstellar Matter, ed. I. Appenzeller, \& C. Jordan, IAU Symp., 122, 81

Bouwman, J., de Koter, A., Dominik, C., \& Waters, L. B. F. M. 2003, A\&A, 401, 577

Brittain, S. D., Najita, J. R., \& Carr, J. S. 2009, ApJ, 702, 85

Calvet, N., D'Alessio, P., Hartmann, L., et al. 2002, ApJ, 568, 1008

D’Alessio, P., Merín, B., Calvet, N., Hartmann, L., \& Montesinos, B. 2005, Rev. Mex. Astron. Astrofis., 41, 61

Dartois, E., Dutrey, A., \& Guilloteau, S. 2003, A\&A, 399, 773

Dent, W. R. F., Greaves, J. S., \& Coulson, I. M. 2005, MNRAS, 359, 663

Dullemond, C. P., \& Dominik, C. 2005, A\&A, 434, 971

Dutrey, A., Guilloteau, S., \& Simon, M. 1994, A\&A, 286, 149

Geers, V. C., Augereau, J.-C., Pontoppidan, K. M., et al. 2006, A\&A, 459, 545

Geers, V. C., van Dishoeck, E. F., Visser, R., et al. 2007, A\&A, 476, 279

Glassgold, A. E., Najita, J., \& Igea, J. 2004, ApJ, 615, 972

Gorti, U., \& Hollenbach, D. 2008, ApJ, 683, 287

Grady, C. A., Polomski, E. F., Henning, T., et al. 2001, AJ, 122, 3396

Greaves, J. S., Mannings, V., \& Holland, W. S. 2000, Icarus, 143, 155

Guilloteau, S., \& Dutrey, A. 1998, A\&A, 339, 467

Guilloteau, S., \& Dutrey, A. 2008, Ap\&SS, 313, 95

Güsten, R., Nyman, L. A., Schilke, P., et al. 2006, A\&A, 454, L13

Henning, T., Burkert, A., Launhardt, R., Leinert, C., \& Stecklum, B. 1998, A\&A, 336,565

Hernández, J., Calvet, N., Briceño, C., et al. 2007, ApJ, 671, 1784

Hillenbrand, L. A. 2008, Phys. Scr. Vol. T, 130, 014024

Hogerheijde, M. R., \& van der Tak, F. F. S. 2000, A\&A, 362, 697

Hollenbach, D. J., Yorke, H. W., \& Johnstone, D. 2000, Protostars and Planets IV, 401
Isella, A., Testi, L., Natta, A., et al. 2007, A\&A, 469, 213

Jonkheid, B., Faas, F. G. A., van Zadelhoff, G.-J., \& van Dishoeck, E. F. 2004, A\&A, 428, 511

Jonkheid, B., Kamp, I., Augereau, J., \& van Dishoeck, E. F. 2006, A\&A, 453, 163

Jonkheid, B., Dullemond, C. P., Hogerheijde, M. R., \& van Dishoeck, E. F. 2007, A\&A, 463, 203

Kamp, I., \& Dullemond, C. P. 2004, ApJ, 615, 991

Kamp, I., Freudling, W., Robberto, M., Chengalur, J., \& Keto, E. 2008, Phys. Scr. Vol. T, 130, 014013

Klein, B., Philipp, S. D., Krämer, I., et al. 2006, A\&A, 454, L29

Koerner, D. W., Sargent, A. I., \& Beckwith, S. V. W. 1993, Icarus, 106, 2

Lecavelier des Etangs, A., Deleuil, M., Vidal-Madjar, A., et al. 2003, A\&A, 407, 935

Malfait, K., Waelkens, C., Waters, L. B. F. M., et al. 1998, A\&A, 332, L25

Nomura, H., \& Millar, T. J. 2005, A\&A, 438, 923

Panić, O., \& Hogerheijde, M. R. 2009, A\&A, 508, 707

Panić, O., Hogerheijde, M. R., Wilner, D., \& Qi, C. 2008, A\&A, 491, 219

Panić, O., Hogerheijde, M. R., Wilner, D., \& Qi, C. 2009, A\&A, 501, 269

Pantin, E., Waelkens, C., \& Lagage, P. O. 2000, A\&A, 361, L9

Pinte, C., Padgett, D. L., Ménard, F., et al. 2008, A\&A, 489, 633

Qi, C., Ho, P. T. P., Wilner, D. J., et al. 2004, ApJ, 616, L11

Qi, C., Wilner, D. J., Calvet, N., et al. 2006, ApJ, 636, L157

Quillen, A. C. 2006, ApJ, 640, 1078

Quillen, A. C., Varnière, P., Minchev, I., \& Frank, A. 2005, AJ, 129, 2481

Raman, A., Lisanti, M., Wilner, D. J., Qi, C., \& Hogerheijde, M. 2006, AJ, 131, 2290

Schöier, F. L., van der Tak, F. F. S., van Dishoeck, E. F., \& Black, J. H. 2005, A\&A, 432, 369

Simon, M., Dutrey, A., \& Guilloteau, S. 2000, ApJ, 545, 1034

Sturm, B., Bouwman, J. Henning, Th., et al. 2010, A\&A, 518, L129

Tannirkulam, A., Monnier, J. D., Harries, T. J., et al. 2008, ApJ, 689, 513

Thé, P. S., de Winter, D., \& Perez, M. R. 1994, A\&AS, 104, 315

Thi, W. F., van Dishoeck, E. F., Blake, G. A., et al. 2001, ApJ, 561, 1074

Thi, W.-F., van Zadelhoff, G.-J., \& van Dishoeck, E. F. 2004, A\&A, 425, 955

van den Ancker, M. E., de Winter, D., \& Tjin A Djie, H. R. E. 1998, A\&A, 330, 145

van der Plas, G., van den Ancker, M. E., Acke, B., et al. 2009, A\&A, 500, 1137 van der Tak, F. F. S., Black, J. H., Schöier, F. L., Jansen, D. J., \& van Dishoeck, E. F. 2007, A\&A, 468, 627

van Kempen, T. A., van Dishoeck, E. F., Brinch, C., \& Hogerheijde, M. R. 2007, A\&A, 461, 983

van Zadelhoff, G.-J., van Dishoeck, E. F., Thi, W.-F., \& Blake, G. A. 2001a, A\&A, 377, 566

van Zadelhoff, G.-J., van Dishoeck, E. F., Thi, W.-F., \& Blake, G. A. 2001b, A\&A, 377, 566

Vieira, S. L. A., Pogodin, M. A., \& Franco, G. A. P. 1999, A\&A, 345, 559

Waelkens, C., Waters, L. B. F. M., de Graauw, M. S., et al. 1996, A\&A, 315, L245

Wilner, D. J., Bourke, T. L., Wright, C. M., et al. 2003, ApJ, 596, 597

Wilson, T. L., \& Rood, R. 1994, ARA\&A, 32, 191

Woitke, P., Kamp, I., \& Thi, W.-F. 2009, A\&A, 501, 383 